


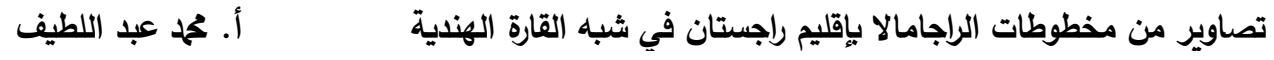

مجلة كلية الآداب بالوادي الجديد - مجلة علمية محكمة- العدد الثالث عشر

\section{تصاوير من مخطوطات الراجامالا بإقليم راجستان في شبه القارة الهندية "ألبوم لاود راجاهالا أنموذجاً"}

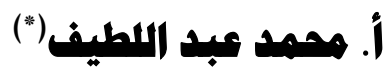

ملخص:

يتناول البحث مخطوط الراجامالا من خلال ألبوم لاود راجامالا بمدرسة التصوير المحلية بيكانير في إقليم راجستان، وتُعد الراجامالا لوحات مستوحاة من الموسيقى والألحان الهندية الأصيلة، التي ازدهرت ما بين القرنين السابع عشر والتاسع عشر الميلاديين، ونفذت تلك اللوحات تحت الرعاية الملكية السامية لأكبر ملوك وشاهات ولايات الهند.

ويهدف البحث إلي دراسة أهمية الراجامالا و تاريخها الأدبي والثعري من خلال النظر في أنماط ألبوم لاود راجامالا الذي يعود مكان تصويره إلي مدرسة بيكانير المحلية بإقليم راجستان، ويحتوي علي مجموعة من التصاوير التي بها معاني وتقسيرات رمزية داخل الراجامالا، وتحليلها، مُتبعا في ذلك المنهج الوصفي التحليلي ومراعياً لعنصر الزمان أي بالترتيب التاريخي من الأقدم إلي الأحدث. الكلمات الدالة: ألبوم - راجامالا -لاود - بيكانير - راجستان. وصف الألبوم: - 2 - 2

تبرع رئيس أساقفة كانتبري "ويليام لاود William Laud"(') بين عامي

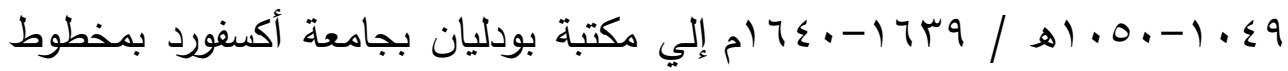
(1) باحث دكتور اه بقسم الآثار بكلبة الآداب- جامعة الو ادي الجديد.

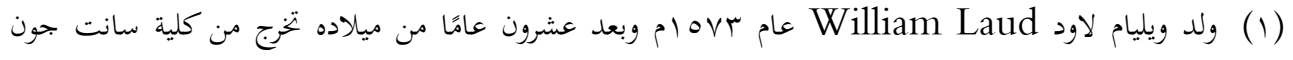

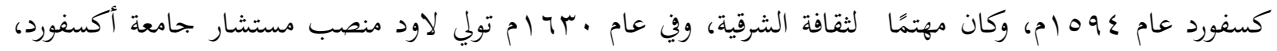

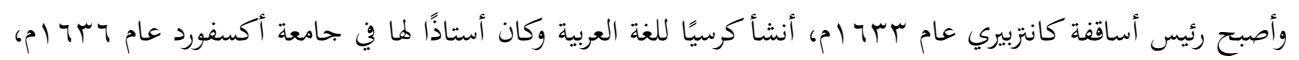

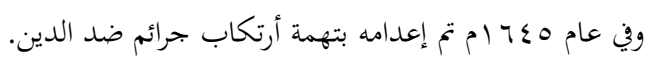
Herbert Johnston Stooke and Karl Khandalavala : The Laud Ragamala Miniatures A Study in Indian Painting and Music, Bruno Cassirer Oxford, 19०ץ, P.^.

$$
\text { (100) }
$$




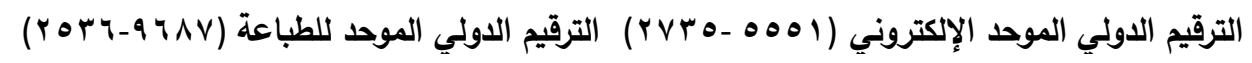

مجلة كلية الآداب بالوادي الجديد- مجلة علمية محكمة- العدد الثالث عشر

MS. " هندي تحت مسمى "لاود رجامالا Laud Ragamala"

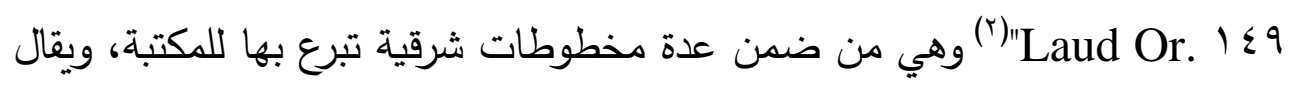
إنها أول مخطوطة هندية تصل إلي مجموعة غربية، وتتكون هذه المخطوطة من فن

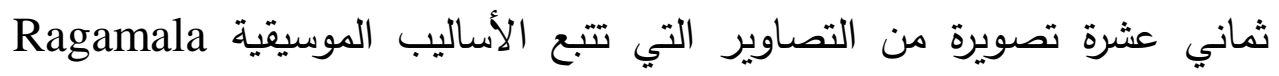

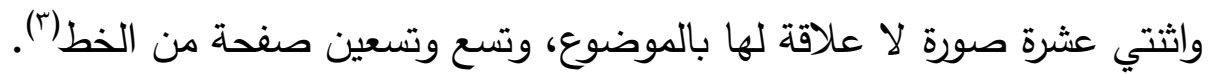

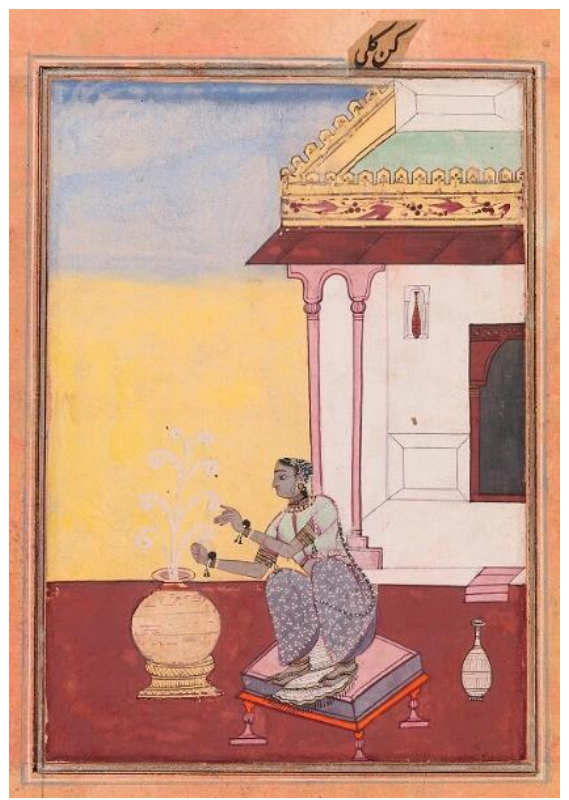

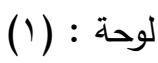
أولاً: الدراسة الوصفية لتصاوير ألبوم لاود راجامالا.

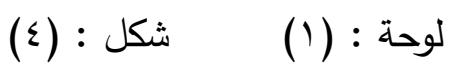
موضوع التصويرة: امرأة تقوم بتحضير أكليل من الزهور . اللحن المستخدم: جنكالي راجيني Gunakali ragini اسم الألبوم: لاود رجامالا.

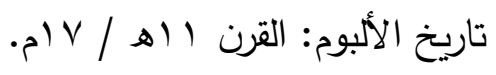

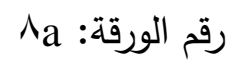

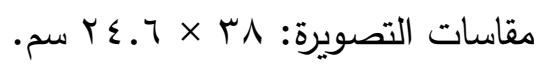
مكان الحفظ: مكتبة بودليان بجامعة أكسفورد.

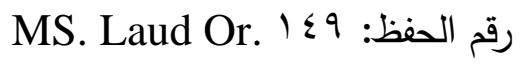
المركز الفني: بيكانير .

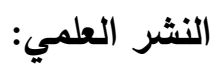

- Molly Aitken: The Laud Ragamala Album, Fig.^.

- Stooke \& Khandalavala: The Laud Ragamala Miniatures, Pl.III الوصف : منظرًا تصويري يمثل امرأة جالسة في ساحة منزلها علي كرسي مربع وأمامها كبيرة، تقوم بجمع إكليل من الزهور تمهيدًا لوصول عشيقها.

( ) Stooke, khandalavala: The Laud Ragamala, P. .

( $r$ ) Molly Emma Aitken: The Laud Ragamala Album Bikaner and the Sociability of Subimperial Painting, Vol. ${ }^{\top}$, No. ', Duke University Press $r \cdot 1 r$, P.r. 
مجلة كلية الآداب بالوادي الجديد - مجلة علمية محكمة- العدد الثالث عشر

جاءت مقدمة التصويرة عبارة عن أرشية باللون الأحمر وضع عليها كرسي لتجلس عليه السيدة وزهرية الزرع وقنينة شراب، أما عن خلفية التصويرة فقد شغلها المصور بجزء من منزل السيدة الذي يظهر من الداخل باللون الأبيض وبه فتحه باب معقودة، والجزء الآخر جاء باللون الأصفر ، وعبر عن الأفق باللون الأزرق الفاتح اشتملت التصويرة من أعلى على كلمة "كن كلي" وترجمتها: "جنكالي Gunakali"

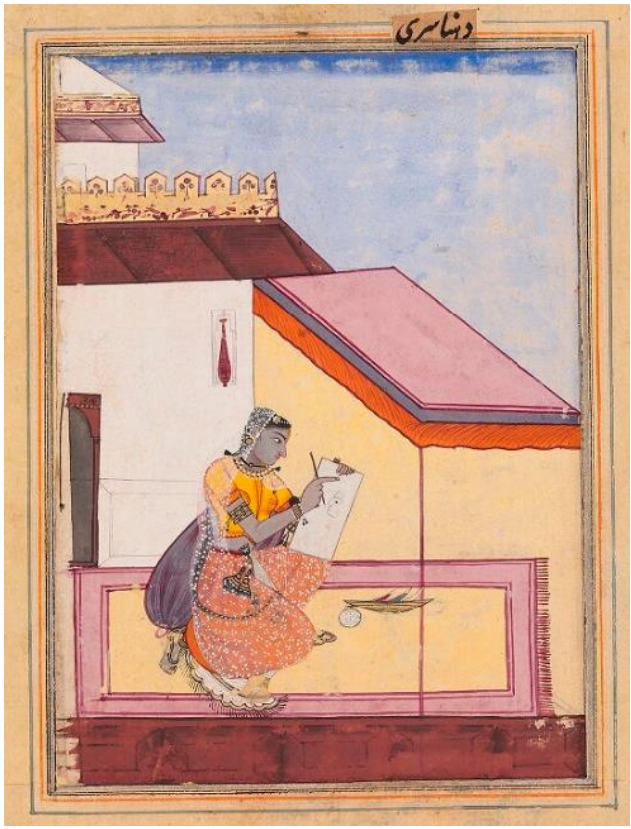

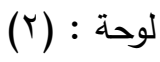
وهو اللحن المستخدم في التصويرة والذي يعبر عن الحالة المزاجيه لها.

لوحة : (r) موضوع التصويرة: امرأة تقوم برسم حبيبها. اللحن المستخدم: دهانشري اجيني Dhanashri ragini اسم الألبوم: لاود رجامالا.

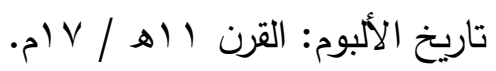

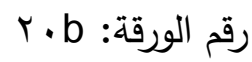

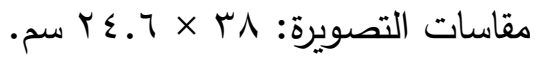
مكان الحفظ: مكتبة بودليان بجامعة أكسفورد.

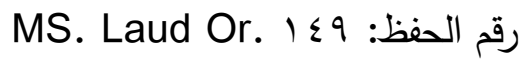
المركز الفني: بيكانير · - الم النشر العلمي:

- Stooke \& Khandalavala: The Laud Ragamala Miniatures, Pl.VIII. الوصف:

تمثل التصويرة منظرًا لامرأة تجلس في ساحة منزلها المكون من طابقين على سجادة بسيطة التكوين يعلوها مظلة تستند بظهرها علي وسادة، تقوم برسم صورة لحبيبها البعيد، أمامها طبق به الألوان. 


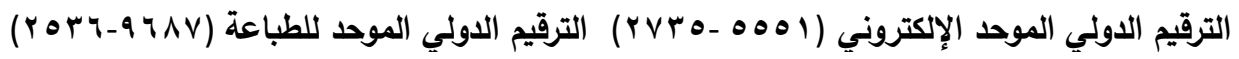

مجلة كلية الآداب بالوادي الجديد - مجلة علمية محكمة- العدد الثالث عشر

تضم خلفية التصويرة جزعًا من واجهة معمارية مكونة من طابقين تمثل منزل السيدة، والجزء الآخر من الخلفية جاء باللون الأصفر، وعبر الفنان عن السماء باللون الأزرق اللبني، حيث لم يوفق الفنان في رسم سور يحيط بساحة المنزل كما هو المعتاد في رسم بيكانير ·

اشتملت التصويرة من أعلى على كلمة ترجمتها: " دهانشري Dhanashri" وهو اللحن المستخدم في التصويرة والذي يعبر عن الحالة المزاجية لها.

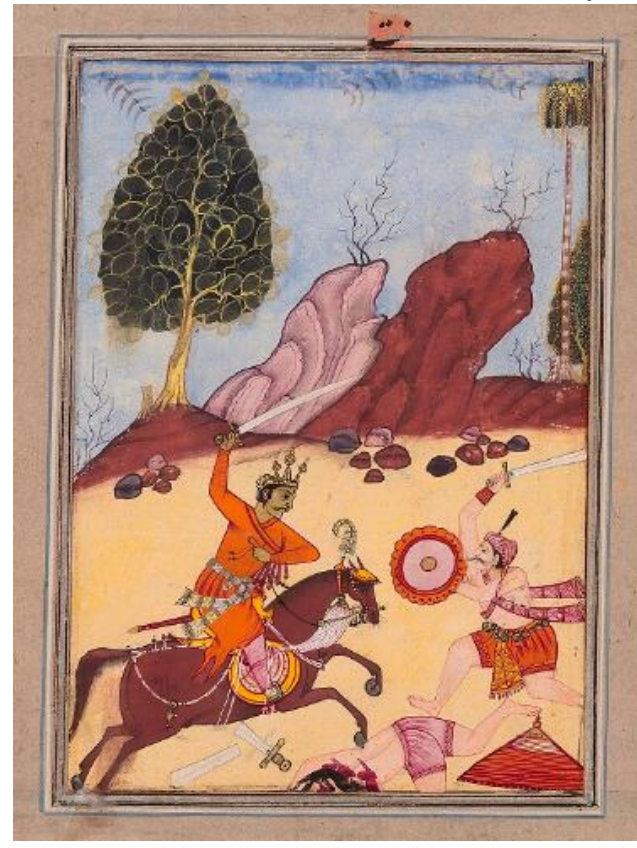

لوحة : (r)

موضوع التصويرة : أمير يقاتل أثنين من جنود المشاه. Nata ragini اللحن المستخدم: ناتا راجيني اسم الألبوم : لاود رجامالا.

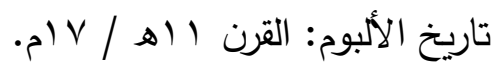
رقم الورقة: roa

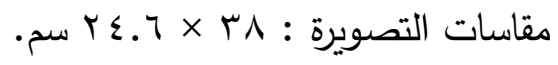
مكان الحفظ: مكتبة بودليان بجامعة أكسفورد.

MS. Laud Or. رقم الحفظ: 9 ـ الجن المركز الفني: بيكانير • - الم

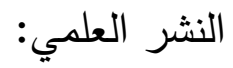
لوحة : (r)

- Stooke \& Khandalavala : The Laud Ragamala Miniatures, P1.IX.

$$
\text { الوصف: }
$$

منظرًا تصويري يمثل فارسًا يمتطي صهوة جوادة المسرج والملجم سيفه في وجه أحد جنود المشاة الذي يحمل درعاً وسيفاً، بينما آخر ملقى على الأرض بعد أن توجهت لله ضربة كسرت سيفه وأسقطته ميتاً، والتصويرة مفعة بالحركة والحيوية سواء بالنسبة للأشخاص أو الخيل المستعمل في القتال. 
تصاوير من مخطوطات الراجامالا بإقليم راجستان في شبه القارة الهنلية أ. محمد عبد اللطيف

مجلة كلية الآداب بالوادي الجليد- مجلة علمية محكم- العدد الثالث عشر

جاءت ملابسهم غير مسلحة أو مدرعة وإن كانوا يحملون السيوف والدروع، فكانت

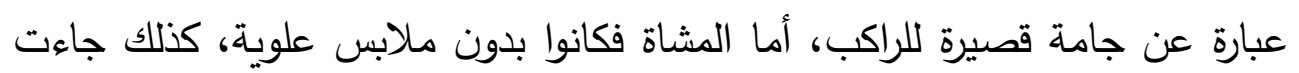
الخلفية عبارة عن أرض جرداء بها صخور خلفها بعض الأثجار، وعبر عن السماء

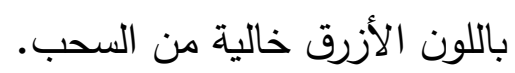

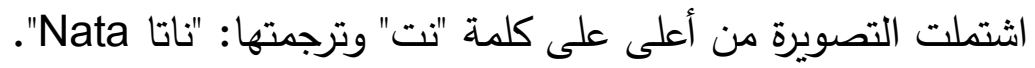

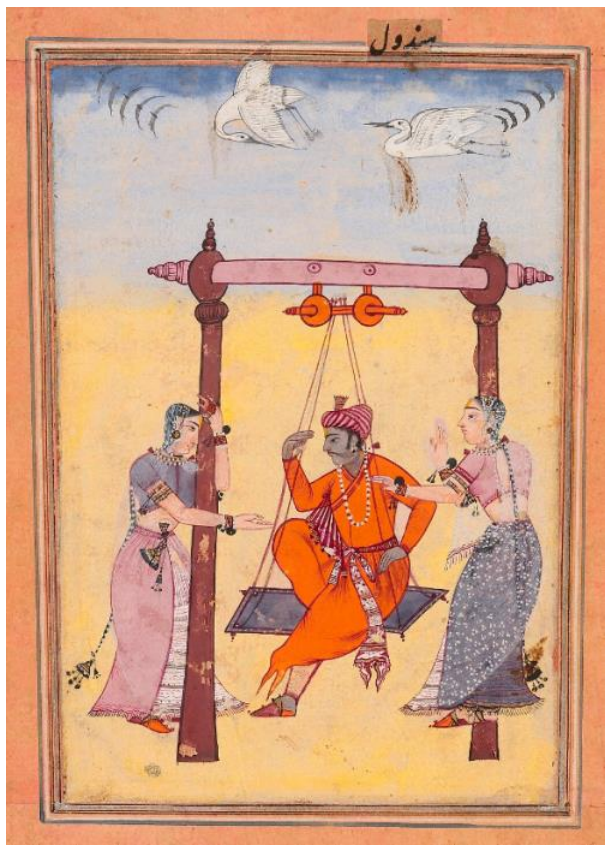

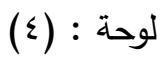

لوحة : (乏) موضوع التصويرة : أمير يجلس علي أرجوحة تدفعها اثثتان من العوانس.

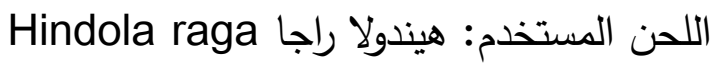
اسم الألبوم : لاود رجامالا.

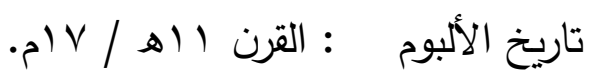

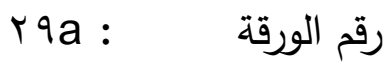

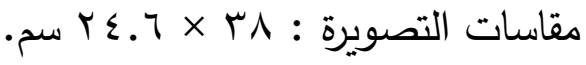
مكان الحفظ : مكتبة بودليان بجامعة أكسفورد.

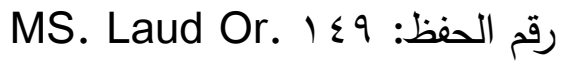
المركز الفني: بيكانير.

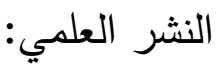

- Molly Aitken: The Laud Ragamala Album, P.r·, Pl.`.

- Stooke \& khandalavala: The Laud Ragamala Miniatures, Pl.X.

$$
\text { الوصف: }
$$

تمثل التصويرة منظرًا لعاشق يجلس علي أرجوحة بينما تقف علي يمينه ويساره

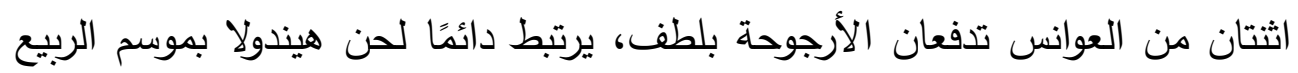

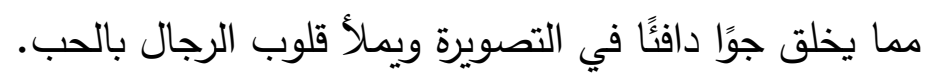




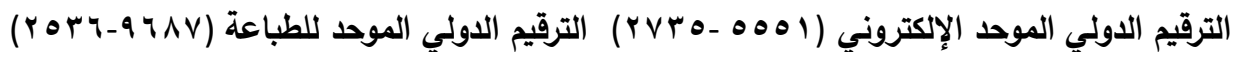

مجلة كلية الآداب بالوادي الجديد- مجلة علمية محكمة- العدد الثالث عشر

نفذت التصويرة علي أرضية باللون الأصفر، وقد عبر الفنان عن الأفق باللون

الأزرق المتدرج تسبح به بعض طيور الكراكي.

اشتملت التصويرة من أعلى على كلمة "ندول"ترجمتها: "هيندولا Hindola" ويعني التأرجح في اللغة السنسكريتية.

ثانيًا: الدراسة التحليلية لتصاوير ألبوم لاود راجامالا.

راجامالا Ragamala

راجامالا Ragamala هي كلمة مترجمة من السنسكريتية وتعني إكليلً من

الألحان الموسيقية، وتتكون من مقطعين؛ (راجاRaga) وهي مشتقة من الجزر

السنسكريتية رانج Rang بمعني المصبوغة أو الملونة أي أن كلمة راجا تدل علي كل ما يلون أو يغلف العقل وصولًا لإرضاء عقول الناس، وقد تمت ترجمتها خطأ بإنها تعني لحنًا أو مفتاحًا، وكلمة (Mala) بمعني إكليل(؟). والراجامالا "نظامًا موسيقيًا متكاملً تتميز فيه كل وحدة من وحداته بتصوير

منظور يرتبط بها وحدها حيث تكون سمة مقابلة عضوية بين اللون والنغم"(ه)

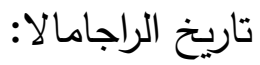

بعد قرون من كتابة الأشكال الموسيقية والشعر، ظهر شكل فني أخر (؟) جمع ثلاثة أشكال للفن وهي الموسيقي والثعر والرسم معًا في لوحة واحدة تعرف باسم راجامالا(V).

(₹) Sudip Ray: Representation of Architecturein in Indian Paintings With Reference to Rajasthani Miniature Paintings, Department of Textile Designing, University of Banasthali Vidyapith, Y. I , P.VI, Nirmala Sharma: A critical study of ragamala paintings of Gujarat Rajasthan and central India, Department of History, Gujarat University 1990, P. IV_rr.

(0) ثروت عكاشة: موسوعة التصوير الإسلامي ، سلسلة ريخ الفن: العين تسمع والأذن تري ، مكتبة لبنان، الطبعه

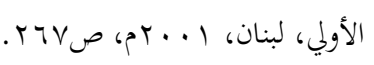

( $\urcorner$ ) Klaus Ebeling: Ragamala Painting, Ravi Kumar, ' st edition 19vץ, P. I ₹. 
مجلة كلية الآداب بالوادي الجديد - مجلة علمية محكمة- العدد الثالث عشر

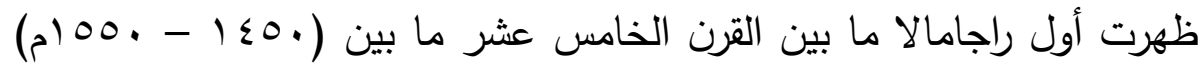

واستمرت في التطور حوالي ثلاثمائة عام حتى توققت في القون التاسع عشر(^)،

وكانت راجامالا هي اللوحات الأكثر شعبية والتي صنفت للرعاة الهندوس من القرن

الخامس عشر وحتى القرن التاسع عشر(9)، وحصلت على مكانة كبيرة حتي أنها

كانت تقدم كمهر وكهدايا رسمية للأثرياء (·').

ظهرت لنا مجموعات مختلفة من هذا المخطوط مشتتة وموزعة حول العالم بين

المتاحف والمجموعات الخاصة، ومن أقدم المجموعات التي وصلت لنا حتي يومنا

هذا علي سبيل الذكر لا الحصر:

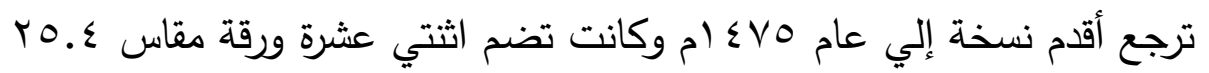

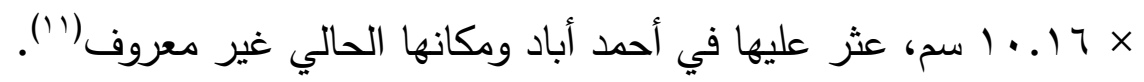

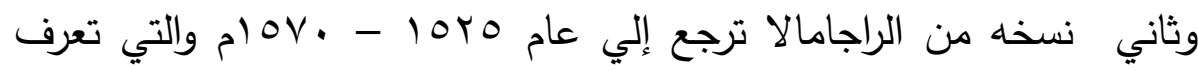

باسم Ragamala suri وهي نسخة غير مكتملة، مقاس أوراقها ع.0 × سم، عثر عليها في ولاية أوتار براديش، والجدير بالذكر أنها كانت تتبع نظام الرسامين في راجستان (r')

هناك نسخة أخري مكونة من ست وثلاثين لوحة في مجموعة بهارت كلي

بهافان ببيناراس Bharat Kala Bhavan, Benaras تؤرخ بعام Ov مام، وجدت

( $\vee$ ) Susan Fuchser: Reflections of Ragamala Painting, thesis or dissertion, Denton, Texas $r \cdots$, P. $r$ r.

( $\wedge$ ) Benjamin Rowland: The Pelican History of Art: The Art and Architecture of India Buddhist, Hindu Jain, Penguin Books I $9 \vee v$, P. $r \varepsilon r-r \varepsilon \varepsilon$. Ebeling: Ragamala Painting, P. I $\leqslant, 1 Y$.

( $\left.{ }^{9}\right)$ Fuchser: Reflections of Ragamala Painting, P. ${ }^{9}$.

( $\cdot$ ) Milo Cleveland Beach: The New Cambridge History of India: Mughal and Rajput Painting Illustrated Edition, Cambridge University Press, Volume ', Part ${ }^{2}$, Y... P. P. .

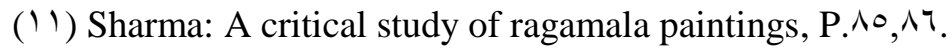

(I ) Sharma: A critical study of ragamala paintings, P.^ฯ.

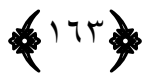


مجلة كلية الآداب بالوادي الجديد- مجلة علمية محكمة- العدد الثالث عشر

في ولاية جوجارات وليس لها تاريخ أو اسم فنان أو منطقة ولكنها اتبعت نظام الرسامين.

وتتسب أقدم راجامالا تنتمي إلي إقليم راجستان في شمال الهند تتتمي لتشاواند

Chawand وهي الآن مبعثرة في مجموعات حول العالم ما بين المتاحف والمجموعات الخاصة،

وفي كل لوحة تم نقش اسم الراجا أو الراجيني باللغة السنسكريتية(rا').

تم العثور علي لوحات راجامالا في أجزاء مختلفة من شبه القارة الهندية، وما

يثير الدهشة هو إيجاد هذا النوع الفريد من الرسم معدًا ومخططًا له من قبل ويتبع نظامًا إن لم يكن موحدًا بشكل كامل إلا أنه لم يخرج عن المألوف بالرغم من أن المخطوط قد رُسم في مناطق متفرقة من البلاد، فلم نجد إلا اختلافات طفيفة في واحدة أو اثنين من التصاوير في كل ألبوم(ع) حيث أتت إلينا نسخ تتمتي إلي ماديا براديش وجوجارات والمناطق البهارية وديكان في الجنوب(10)، كذلك في إقليم راجستان في ماروار Marwar - سيروهي Sirohi - أودييور Uduipu - - بالي Paliجودبور Bodhpur - Bundi مota - كونديكانير - Bikaner - يونيارا

Malpura مالبورا - Uniara

Raga and Ragini الراجا والراجيني

تُعرف الراجا علي أنها تركيبة معينة أو علاقة العديد من النغمات التي ترضي

$$
\text { الأذن، وهو التعريف الوارد في الكتب السنسكريتية(ז'). }
$$

ويعرفها أيضًا أنها عبارة عن تلوين موسيقي، يرتبط هذا التلوين بمزاج أو شعور

أو عاطفة معينة، غالبًا ما ترتبط هذه العاطفة بوقت محدد من اليوم('vنا.).

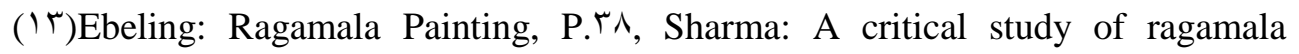

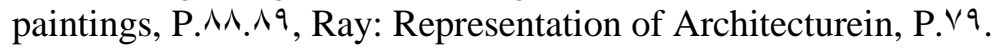

( 1 ) Sharma: A critical study of ragamala paintings, P. YY.

(10)Sharma: A critical study of ragamala paintings, P. r r.

( 1 7) O.C.Ganguly: Ragas and Raginis, Nalanda Books, 1940, P. . . 
مجلة كلية الآداب بالوادي الجديد - مجلة علمية محكمة- العدد الثالث عشر

جاءت الراجا والراجيني في شكل عائلات حيث مُثلت الراجا علي أنهم الذكر ولكل منهم مجموعة من الأزواج (الراجيني) ومن الجدير بالذكر يُعد تتبع هذه الأشكال

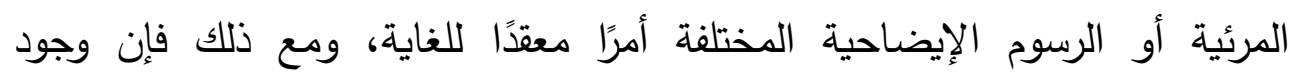
النصوص والأيات المنقوشة باللغة السنسكريتية نستطيع من خلالها معرفة انتماء كل الإنل لوحة إلي اي من الأزواج والعائلات. ولقد اختلفت أنظمة الراجامالا باختلاف السنين والبلاد والمؤلفين، وحاول العديد على مدار السنين تصنفيف تلك الراجا وعائلاتهم، ومن بين الكثير من التصنيفات

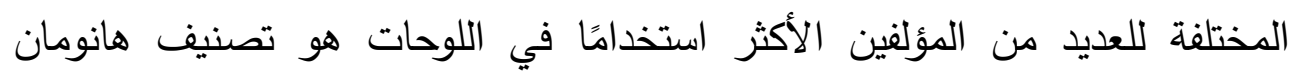
Hanuman Ebling نظام الرسامين Painter's System والأخير أصله غامض ولا ينتمي لأي

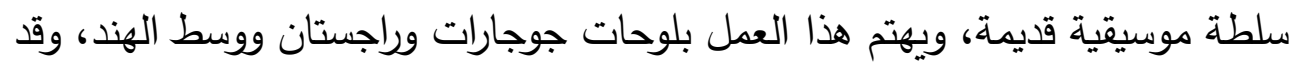
لوحظ ان نظام الرسامين قد استخدم في عدد كبير من اللوحات في هذه المناطق (^)'.

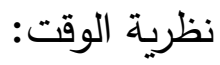

ونظرية الوقت في هذا الموضوع مهمة حيث تتغير العواطف مع مرور الوقت

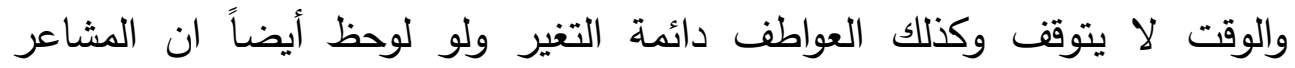
الإنسانية لا تبقى ثابتة ، بل إنها تتغير أيضاً، فجاءت بعض دوالعه الراجا تغني في ساعات

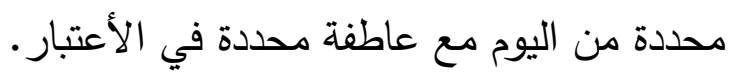

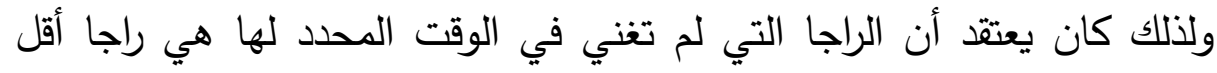

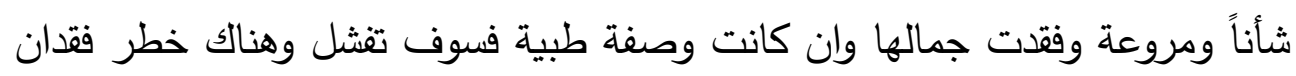

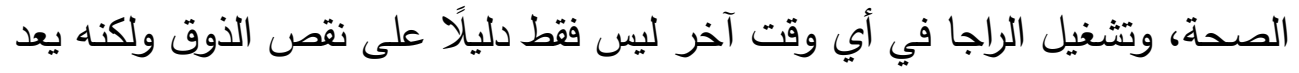

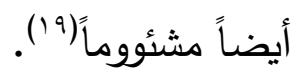

( $\mathrm{V}$ ) Ganguly: Ragas and Raginis, P.०.

( $\wedge$ ) Sharma: A critical study of ragamala paintings, P. $7 \varepsilon$.

(19) Sharma: A critical study of ragamala paintings, P.०. 


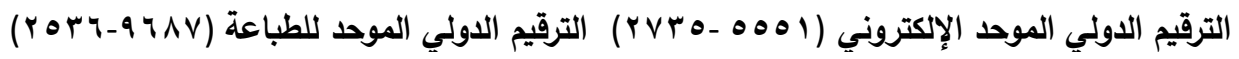

مجلة كلية الآداب بالوادي الجليد- مجلة علمية محكم- العدد الثالث عشر

والفكر الهندوسي يقسم اليوم إلي ساعات محددة ولكل راجا ثلاث ساعات

وشكلت الراجا وفقاً للوقت المحدد (r.) وهم علي النحو التالي:

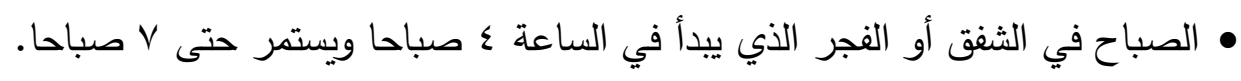

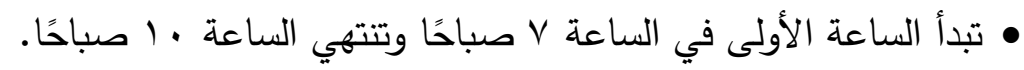

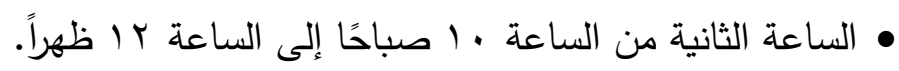

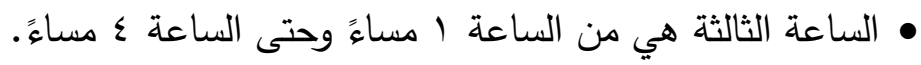

• ساعات المساء الثفق هي من ع مساء إلى V مساء.

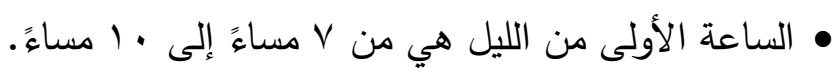

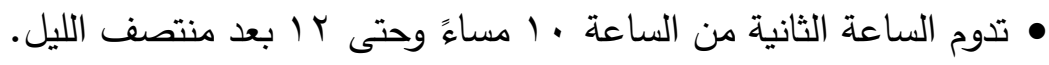

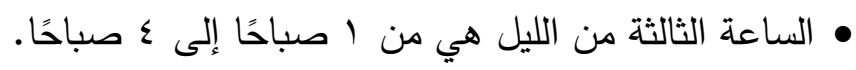

النتائج:

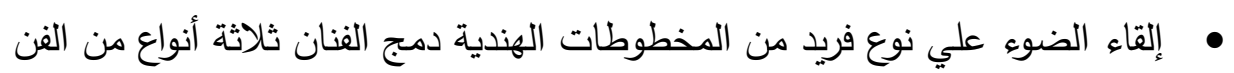

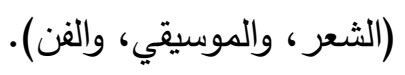

• أوضحت الدراسة أنظمة الراجامالا التي تكونت من عائلات بها ذكور وإناث (راجا

$$
\text { وراجيني) وأبناءهم. }
$$

• توضيح أهية الوقت بالنسبة للراجامالا والعلاقة بينها وبين أشكالها الموسيقية.

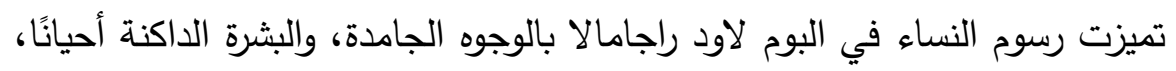

$$
\text { والرؤوس المسطحة، بينما جاءت الرجال ذات بنية قوية، وشوارب مربعة. }
$$

$(\uparrow \cdot)$ Fatima Hina: A comparative Study of Colour and Form in Deccan an Rajasthan Raagmala Painting, Department of Fine Arts Aligarh Muslim University Aligarh, India Y.. 9, P. 9 . 


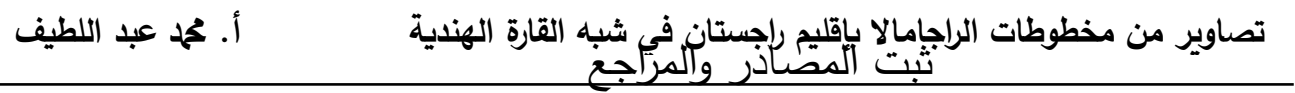

أولاً: المراجع العربية: مجلة كلية الآداب بالوادي الجليد- مجلة علمية محكمة- العدد الثالث عشر

ثروت عكاشة: موسوعة التصوير الإسلامي ، سلسلة تاريخ الفن: العين تسمع والأذن تري ، مكتبة

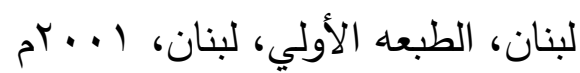

ثانياً: المراجع الأجنبية:

- Benjamin Rowland: The Pelican History of Art: The Art and Architecture of India Buddhist, Hindu Jain, Penguin Books 19vV.

- Fatima Hina: A comparative Study of Colour and Form in Deccan an Rajasthan Raagmala Painting, Department of Fine Arts Aligarh Muslim University Aligarh, India r. . 9

- Herbert Johnston Stooke and Karl Khandalavala : The Laud Ragamala Miniatures A Study in Indian Painting and Music, Bruno Cassirer Oxford, 190r.

- Klaus Ebeling: Ragamala Painting, Ravi Kumar, ' st edition 19Vr.

- Milo Cleveland Beach: The New Cambridge History of India: Mughal and Rajput Painting Illustrated Edition, Cambridge University Press, Volume ', Part $r, r \cdots \wedge$.

- Molly Emma Aitken: The Laud Ragamala Album Bikaner and the Sociability of Subimperial Painting, Vol. ${ }^{7}$, No. ', Duke University Press $r \cdot 1 r$.

- Nirmala Sharma: A critical study of ragamala paintings of Gujarat Rajasthan and central India, Department of History, Gujarat University 1990.

- O.C.Ganguly: Ragas and Raginis, Nalanda Books, 1940.

- Sudip Ray: Representation of Architecturein in Indian Paintings With Reference to Rajasthani Miniature Paintings, Department of Textile Designing, University of Banasthali Vidyapith, Y . 17.

- Susan Fuchser: Reflections of Ragamala Painting, thesis or dissertion, Denton, Texas $r \cdots$.

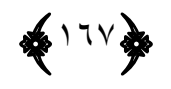

